\title{
Proyectos del IAPH \\ Los datos a examen: estadística e índice de perdurabilidad de los sitios arqueológicos andaluces
}

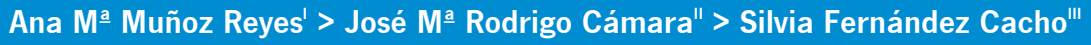

\section{Resumen}

La evaluación de los datos arqueológicos disponibles para la elaboración de un modelo predictivo para Andalucia desde el punto de vista de su tratamiento estadistico ha sido una de las tareas prioritarias en el marco del proyecto MAPA (ver páginas anteriores). Del conocimiento preciso de la calidad de la información de partida puede depender la eficacia del modelo resultante, por lo que la metodologia de trabajo global debe establecerse teniendo en cuenta los resultados obtenidos tras dicha evaluación. En este trabajo se analizan fundamentalmente los datos arqueológicos relacionados con el estado de conservación de los sitios arqueológicos andaluces, como apoyo a la elaboración de un indice de perdurabilidad arqueológica para el conjunto de la comunidad autónoma.

\section{Palabras clave}

Patrimonio arqueológico

Andalucía

Índice de perdurabilidad

Estadistica

Modelos predictivos

\section{Introducción}

Las fuentes de datos fundamentales para la realización de modelos predictivos son, por una parte, las que proporcionan información sobre las características ambientales con incidencia en la distribución de sitios arqueológicos y, por otra, la información arqueológica disponible sobre el área objeto de estudio.

En el caso del Modelo Andaluz de Predicción Arqueológica (MAPA), la información arqueológica de partida está registrada en el Sistema de Información del Patrimonio Histórico (SIPHA). Este sistema contiene información de más de 12.000 localizaciones arqueológicas que han sido documentadas de forma sistemática desde 1985, con diversos objetivos. Entre ellos destaca la realización de un Inventario de Yacimientos Arqueológicos por parte de la Dirección General de Bienes Culturales (Consejería de Cultura, Junta de Andalucía) y, en menor medida, revisiones de información para su catalogación genérica, prospecciones arqueológicas, ejecución de proyectos de documentación arqueológica de áreas concretas llevadas a cabo por el Instituto Andaluz del Patrimonio Histórico, etc. (Mondéjar, 2002).

La diversidad de objetivos y características de los proyectos que han servido para recopilar esta información arqueológica adaptándose al formato normalizado de una única base de datos para el conjunto de la comunidad autónoma, hace necesario su análisis para intentar determinar la calidad y viabilidad de su tratamiento estadístico.

Por este motivo se ha encargado en el último año al Departamento de Estadística e Investigación Operativa de la Universidad de Sevilla la redacción de dos dictámenes técnicos (Muñoz Reyes, 2003; Muñoz Reyes-Rodrigo, 2004). En el primero de ellos se analizaron las tablas de datos que resultaban de mayor interés para la realización del proyecto MAPA (Modelo Andaluz de Predicción Arqueológica) y en el segundo el trabajo se centró en el análisis de aquellos campos de información relacionados con el estado de conservación de los sitios arqueológicos, intentando determinar su potencial informativo para la elaboración de un Indice de Perdurabilidad del Patrimonio Arqueológico, tal y como ha quedado definido en el trabajo precedente de este mismo Boletín.

\section{Calidad de la información disponible para la ejecución del proyecto MAPA desde el punto de vista de su tratamiento estadístico}

Los objetivos fundamentales del primer dictamen técnico de caracter estadístico para el proyecto MAPA (Muñoz Reyes, 2003) se resumen en: 


\section{2 - 073 \\ Proyectos del IAPH}

Los datos a examen: estadística e IP de los sitios arqueológicos andaluces

\section{PH49 - Julio 2004}

I Departamento de Estadística e Investigación Operativa. Universidad de Sevilla
"I Departamento de Estadística e Investigación Operativa. Universidad de Sevilla
III Instituto Andaluz del Patrimonio Histórico

\section{Porcentajes en función de A, B, C y D}

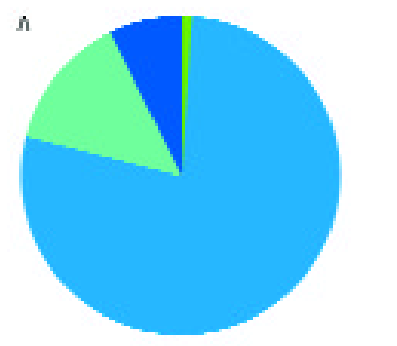

8
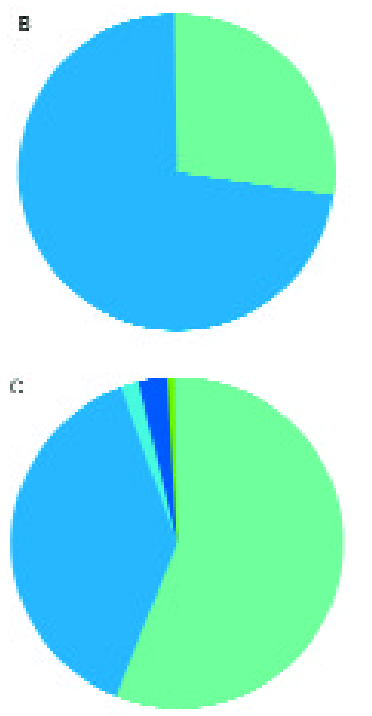

o

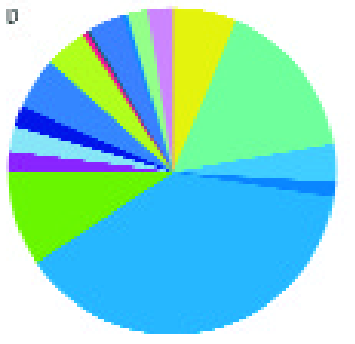

A. Grado de conservación,

B. Causas del deterioro.

Causas genéricas,

C. Causas del deterioro.

Agentes naturales

D. Causas del deterioro.

Agentes humanos

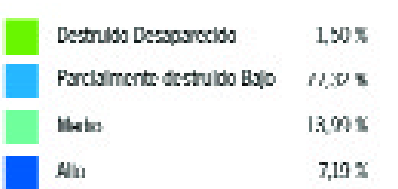

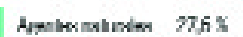

Noorteh hum $22,4 \mathrm{x}$

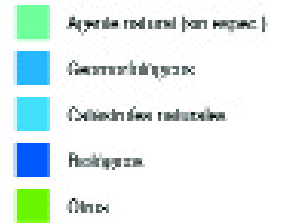

$52,7 x$

\begin{tabular}{|c|c|}
\hline \multicolumn{2}{|l|}{ Dpollosupstit:sl } \\
\hline 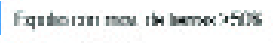 & \\
\hline Nado suboolder & \\
\hline Lsboves tretstes: & \\
\hline 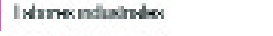 & \\
\hline Draposere & \\
\hline Lxan. arued in coreenasion & \\
\hline Onta & \\
\hline Ueses mitarss & \\
\hline Aemportientos & \\
\hline 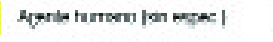 & \\
\hline 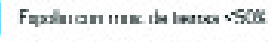 & \\
\hline hodosupicis & \\
\hline Puesta en rege & \\
\hline Actiutsoses erfactios & \\
\hline Oteex puttorex & \\
\hline Nhes irivedes & \\
\hline velas & \\
\hline Actudsdes pecuarts & \\
\hline Imemstrin & \\
\hline
\end{tabular}

> Adecuación de las tablas de datos de DatARQUEOS a los requerimientos de un análisis estadístico.

$>$ Estudio de la fiabilidad de los análisis estadísticos realizados de forma exploratoria en el IAPH, y procedimientos apropiados para la elaboración del modelo.

Un primer análisis realizado sobre la base de datos permitió ofrecer una visión global de la información contenida en las tablas de datos, así como detectar posibles incongruencias, como valores incompatibles o valores perdidos. Los valores incompatibles aparecen cuando se ha introducido un dato que no concuerda con los valores posibles de una tabla de opciones cerradas dependientes de otra variable. Por ejemplo, se introduce 'Epoca Romana' como periodo histórico genérico y 'Edad del Cobre' como periodo histórico específico en un registro asociado a un sitio arqueológico. El valor esperado para el campo de periodo histórico genérico era, en este caso, 'Prehistoria Reciente'. Una vez detectadas, se ha revisado la información completa de los registros que contenian este tipo de incongruencias para corregirlas antes de proceder al análisis estadístico. Por otra parte, aquellos valores correspondientes a variables numéricas que aparecen en blanco son considerados valores perdidos del sistema. Realmente se corresponden con casos en los que no se tiene información del valor de la variable, es decir, deben ser recodificados como 0 con la etiqueta 'no se dispone de información'.

Posteriormente se han realizado unos análisis descriptivos básicos de las principales tablas de la base de datos que han proporcionado las frecuencias absolutas, porcentajes y porcentajes acumulados de cada uno de los valores presentes en cada variable contenida en ellas. Dichos análisis han sido especialmente útiles para determinar la fiabilidad de algunas aproximaciones estadísticas realizadas previamente a este estudio (Fernández Cacho-MondéjarDíaz Iglesias, 2002), y sus resultados más destacables son:

1. Los valores incompatibles y los valores perdidos no influyeron significativamente en los resultados de los análisis realizados. 2. Se establecieron análisis más pormenorizados de las variables numéricas además de la media aritmética (desviación típica, varianza, rango, intervalos de confianza, amplitud, amplitud intercuantil, etc.), que han refinado los resultados entonces presentados.

\section{Los datos de conservación. Viabilidad de uso para la elaboración del Índice de Perdurabilidad de los sitios arqueológicos andaluces}

La información objeto de análisis

Como ya se ha apuntado anteriormente, un análisis estadistico preliminar de carácter descriptivo de algunas de las variables explicativas de la distribución de entidades arqueológicas (en ade- 
lante EAs) en Andalucia sirvió para ofrecer una visión de conjunto de su influencia en forma de tablas y cartografía (Fernández Cacho, Mondéjar y Díaz Iglesias, 2002). Del mismo modo, se fijaba en el trabajo citado el concepto de perdurabilidad que integraba aquellas variables que inciden en el grado de conservación del Patrimonio Arqueológico, sobre todo en la posibilidad de que hayan podido intervenir en su destrucción. También advertía entre otras cuestiones- sobre aspectos cruciales para el desarrollo del proyecto como pueden ser el grado de precisión de las fuentes de información o el nivel de reconocimiento territorial -los denominados "indicadores de conocimiento"-, llegándose a concluir la intrinseca variabilidad de las fuentes empleadas para cumplimentar cada registro a lo que se sumaba la propia naturaleza de la revisión de la información como proceso continuo.

Teniendo en cuenta estas premisas iniciales, se optó por continuar el trabajo estadístico valorando la significación de la información registrada en DatARQUEOS referida al grado de conservación y las causas del deterioro de los sitios arqueológicos documentados. De este modo se trabajaba en una doble vía metodológica: por una parte realizando inferencias estadisticas de los datos existentes y, por otra, elaborando un índice de perdurabilidad hipotético a partir de variables territoriales (Márquez-Vallejo, 2004), para cruzar posteriormente los resultados obtenidos por ambos procedimientos.

En la base de datos la información registrada se encuentra normalizada y se ha codificado tal y como queda reflejado en los gráficos A, B, C y D (Figura 1).

A pesar de que se han incorporado también campos con formato memo para especificar las afecciones producidas en cada entidad arqueológica, la información en este apartado de conservación en la base de datos es muy escasa. Además, las opciones relacionadas con el campo "grado de conservación" han respondido a criterios diversos en función de las fuentes de información empleadas y, al referirse a las entidades en su conjunto, no se distingue, por ejemplo, entre posibles deterioros puntuales o entre grados de conservación distintos de partes diferentes.

Sin embargo, a pesar de las deficiencias que presenta la información, se han realizado diversos análisis sobre ella para detectar posibles correlaciones con otras variables:

$>$ Grado de conservación en función de la clasificación crono-tipológica de las EAs.

$>$ Grado de conservación y causas de deterioro en función de los usos del suelo.

$>$ Grado de conservación y causas de deterioro en función del nivel de protección de las EAs por su propia inclusión en el Catálogo General del Patrimonio Histórico de Andalucia como por su ubicación espacios naturales protegidos.
2. Distribución de registros según el criterio crono-tipológico
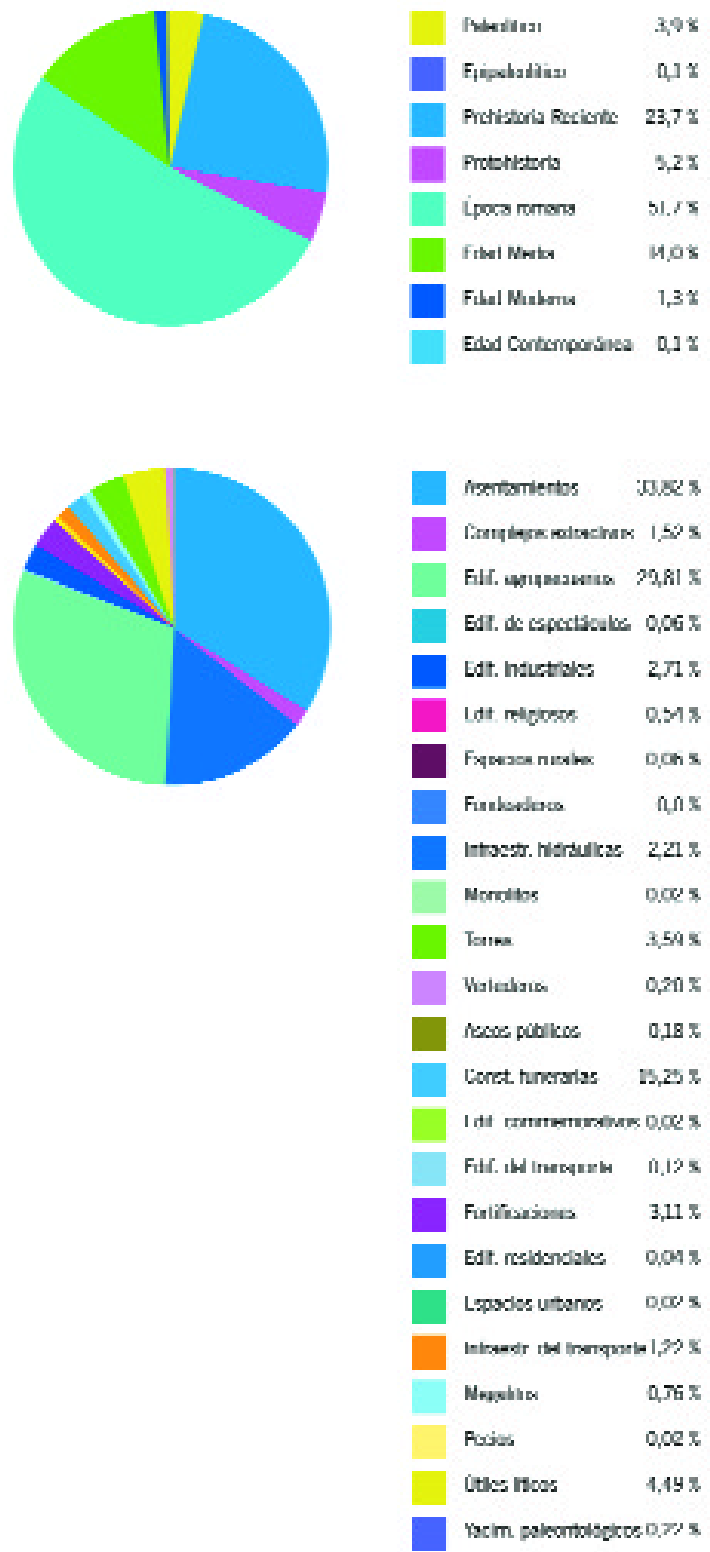

Si las correlaciones estadisticas entre las principales variables seleccionadas por su influencia en el estado de conservación del Patrimonio Arqueológico es positiva, el grado de asociación entre ellas permitirá su mayor o menor aprovechamiento en el diseño del modelo.

\section{Correlaciones estadísticas}

Tras una primera fase del proyecto en la que se trato de manera específica la información arqueológica disponible desde el punto de vista de su análisis estadístico (Muñoz, 2003), en la fase actual se han acometido los análisis cruzados entre las variables tal como se ha expuesto en el apartado anterior. 


\section{$074-075$ \\ Proyectos del IAPH}

Los datos a examen: estadística e IP de los sitios arqueológicos andaluces

\section{PH49 - Julio 2004}

\section{Mapa de las entidades arqueológicas a partir de los niveles de agresividad}

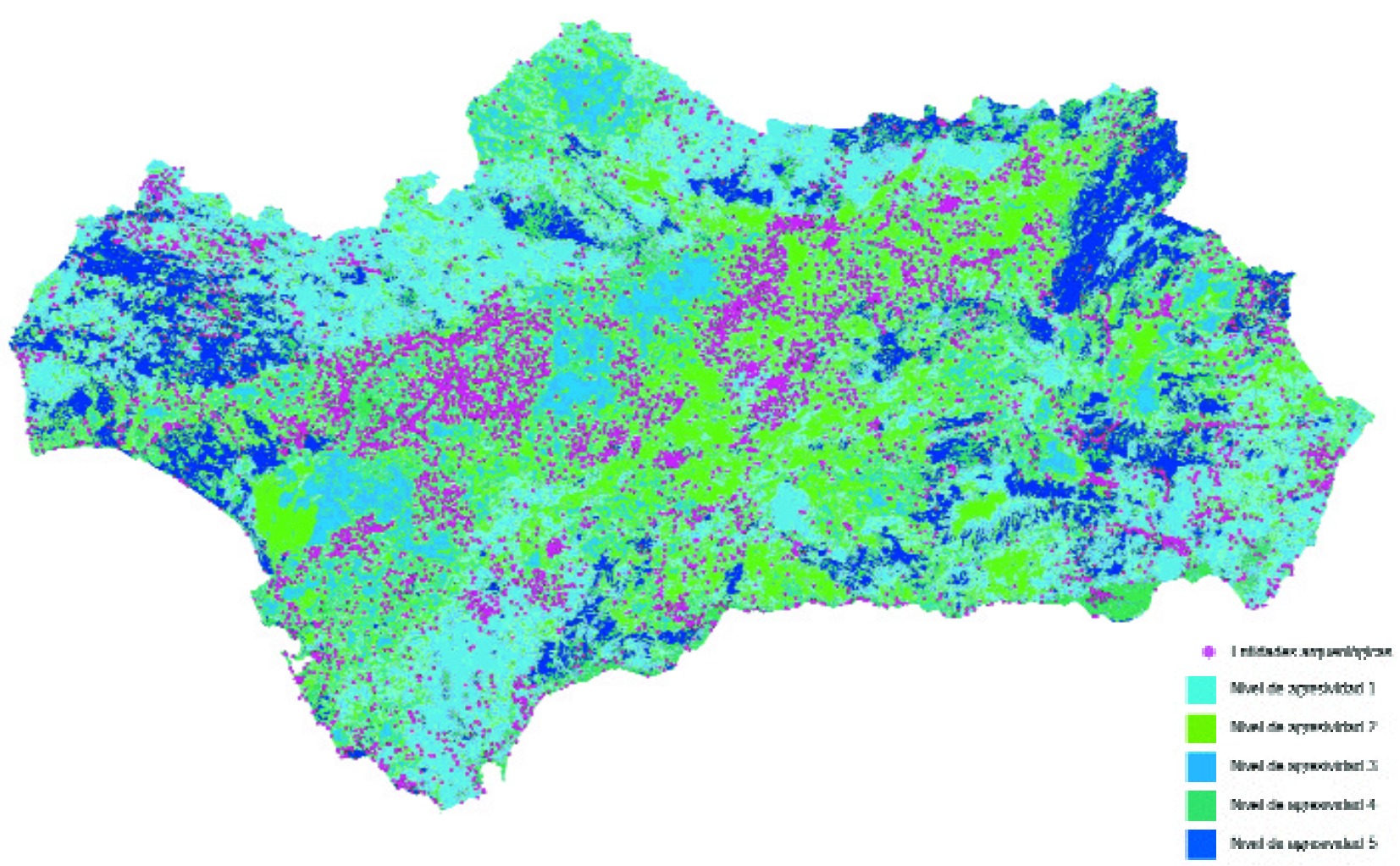

Desde el punto de vista arqueológico conviene introducir algunas precisiones sobre los resultados estadísticos obtenidos, para favorecer una mejor comprensión del hilo argumental que se seguirá:

$>$ En el estudio de la dependencia estadistica entre las variables el método del $X^{2}$ (chi-cuadrado) demuestra su utilidad. Su valor informará sobre si las variables cruzadas son dependientes o independientes, sin embargo el test no informará sobre la intensidad de esa asociación. Para ello se observará el valor del índice de $\phi^{2}$ (phi-cuadrado) en el que valores más cercanos a 0 indican la debilidad de la relación, y el valor máximo que puede obtenerse (1 para tablas $2 \times 2 \circ 2 \times n$, o mayor que 1 para tablas $>=$ a $3 \times 3$, en este último caso $\phi^{2}$ se regula por el índice obtenido por el $V$ de Cramer $^{1}$ ) ofrecerá la relación perfecta o asociación absoluta.

> Los análisis anteriores, no informarán en modo alguno sobre el problema de la causalidad. Una línea para adentrarnos en este terreno es, en primer lugar, hacernos la pregunta de si desaparece o se mantiene una relación entre dos variables cuando introducimos una tercera.

$>$ Volviendo a la interpretación de los resultados que ahora interesan, habría que apuntar que el test de $X^{2}$, debido a su comportamiento respecto al tamaño de la muestra -en este caso se considera como de gran tamaño-, puede proporcionar fácilmente relaciones significativas entre las variables. La dificultad radicara en distinguir entre significación estadística y significación sustantiva (Shennan; 1992: 86). Así pues, el examen a detalle de los datos presentados en una clasificación cruzada es entonces de gran importancia.

Los valores de chi-cuadrado expuestos en la tabla 1, de acuerdo con el bajo valor -no apreciable- de la significación (valores bajos se estiman $<0,05$ por convención), muestran que hay dependencia entre las variables cruzadas, es decir, se rechaza la hipótesis nula que enuncia que las variables son independientes.

Por otra parte, la medida de esta relación (parte derecha de la tabla) muestra la débil asociación que existe entre estas mismas variables. Quedan lejos del valor 1 que indicara la medida óptima de asociación, con lo cual no sería aceptable predecir el valor de una variable a partir de la otra, o al menos se cometerían muchos errores.

Grado de conservación en función de la clasificación crono-tipológica de las EAs

Interesa destacar que su distribución por periodos históricos manifiesta el peso de las categorías "época romana" y "protohistoria" con un $51,7 \%$ y $23,7 \%$ del total de registros respectivamente (Figura 2). Las pruebas realizadas han aportado una medida de la asociación muy baja respecto a la variable "estado de conservación", de hecho desaconseja cualquier inferencia predictiva sobre el estado de conservación de las EAs por periodos históricos. 


\begin{abstract}
${ }^{1}$ En definitiva el indice $V$ de Cramer lo que hace es estandarizar el phi-cuadrado para aquellas tablas mayores a $2 \times 202 \times \mathrm{n}$. Asi pues, el valor máximo que podrá tomar este indice por la fórmula de Cramer será de nuevo 1 , por lo que podremos utilizarlo como un phi-cuadrado regulado.

${ }^{2}$ Esta observación puede hacerse extensible a la variable del apartado siguiente referido a la clasificación tipológica de las EAs.

${ }^{3}$ Mapa de Usos y Coberturas Vegetales del Suelo (1999) editado por la Consejería de Medio Ambiente de la Junta de Andalucía.
\end{abstract}

\section{Gráficos del nivel de agresividad}

A. Grado de conservación

B. Causas genéricas de deterioro
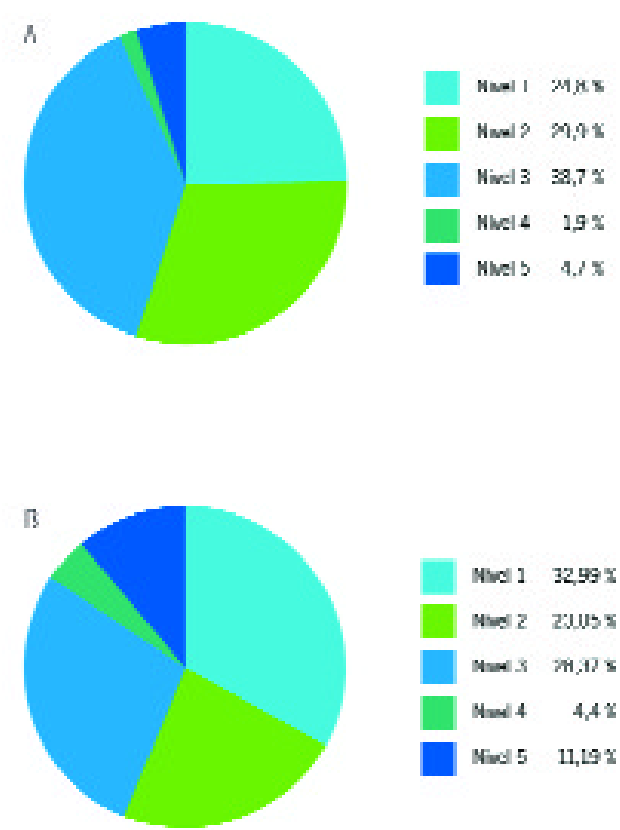

Conviene destacar asimismo que la asignación del "estado de conservación" en la base de datos solo implica a la porción de EAs visible por el informador y por tanto no a todos los valores de "periodo histórico" que puedan asignarse a una misma EA en el momento del inventario. Por tanto la muestra siempre será acotada y hay que tener seguridad sobre a qué periodo histórico de la EA se le está valorando el estado de conservación para preservar la fiabilidad de la muestra². Por ello, se ha optado por contrastar los resultados producidos con los que se obtienen procesando únicamente los datos de conservación de aquellas EAs asociadas a un único periodo histórico, siendo ambos muy similares.

En cuanto a la distribución por tipologías, se observan frecuentes vacios de ocurrencias constatadas para algunas categorias, mostrándose el peso alcanzado por "asentamientos", "construcciones funerarias" y "edificios agropecuarios". Quizás, siendo una clasificación genérica de tipología de sitios arqueológicos, esta variable haya de ser redefinida acotando el número de categorías o reagrupando algunas de las existentes.

Del comportamiento estadístico de estas dos variables anteriores respecto a "estado de conservación" se desprende en primer lugar que no existe independencia entre ellas, es decir, el análisis del chi-cuadrado descarta la hipótesis nula y establece su relación. Sin embargo, las medidas de la asociación de 0,086 y
0,171 respectivamente, no permiten concluir que a determinado periodo histórico o tipología de EA pueda corresponder una tendencia hacia un estado de conservación concreto.

\section{Grado de conservación y causas de deterioro en función} de los usos del suelo

Para analizar las posibles correlaciones entre estas variables se ha procedido, en primer lugar, a la codificación de los distintos usos del suelo ${ }^{3}$ (112 clases originales) en función del grado de agresividad hipotético sobre el Patrimonio Arqueológico (Muñoz Reyes-Rodrigo, 2004).

La escala de valores asignada se ha determinado en función del daño que para la integridad de los restos arqueológicos se le pueda suponer a cada uso en un rango comprendido entre 1 (menor agresividad) y 5 (mayor agresividad) (Figuras 1,3 y 4).

Los resultados del análisis cruzado con la variable "estado de conservación" reflejan una medida de la asociación muy baja $(0,070)$ que imposibilita la predicción del comportamiento de una respecto a la otra (Figura 5). Igualmente, la representación del cruce recíproco, es decir, la distribución de la variable "nivel de agresividad" para cada categoría de "estado de conservación" solo refleja una débil variación (Figura 6) en el valor de "parcialmente destruido-bajo".

Respecto al análisis cruzado de las causas de deterioro provocadas por agentes humanos y/o naturales, se destaca una medida de la asociación algo más representativa $(0,199)$. Teniendo en cuenta la asignación del nivel 1 a usos del suelo tradicionalmente alejados del manejo humano intensivo (pastizales y matorrales en sus distintas variantes, dunas y arenales...), se puede comprobar que lo obtenido es consistente respecto a una esperable "ausencia" o mínimo impacto de agentes humanos en las áreas pertenecientes a este nivel de agresividad. El mismo tipo de razonamiento puede utilizarse para explicar lo observado en el nivel 3, donde se advierte un mayor impacto del agente humano como causa genérica de deterioro, ya que este nivel se asocia con las áreas donde el manejo humano es más intensivo sobre el territorio (superficies agricolas de herbáceos-leñosos en regadio, olivar-viñedo y sus mezclas).

En relación con las causas del deterioro producidas por agentes naturales se observa también una medida de la asociación muy baja $(0,117)$. La explicación de este comportamiento puede ser que el nivel de agresividad sobre el que se ha realizado el análisis procede de una evaluación de usos antrópicos que no tiene en cuenta especialmente la incidencia de otros posibles agentes naturales que podrian afectar a la conservación de las EAs.

Por último, respecto a las causas de deterioro por agentes humanos en función de los niveles de agresividad, la medida de asociación $(0,23)$ resultante es la más alta en el contexto de debilidad general observado (Tablas 1 y 2. ver anexos). Un segun- 
$076-077$

\section{Proyectos del IAPH}

Los datos a examen: estadística e IP de los sitios arqueológicos andaluces

\section{PH49 - Julio 2004}

5. Grado de conservación por niveles de agresividad
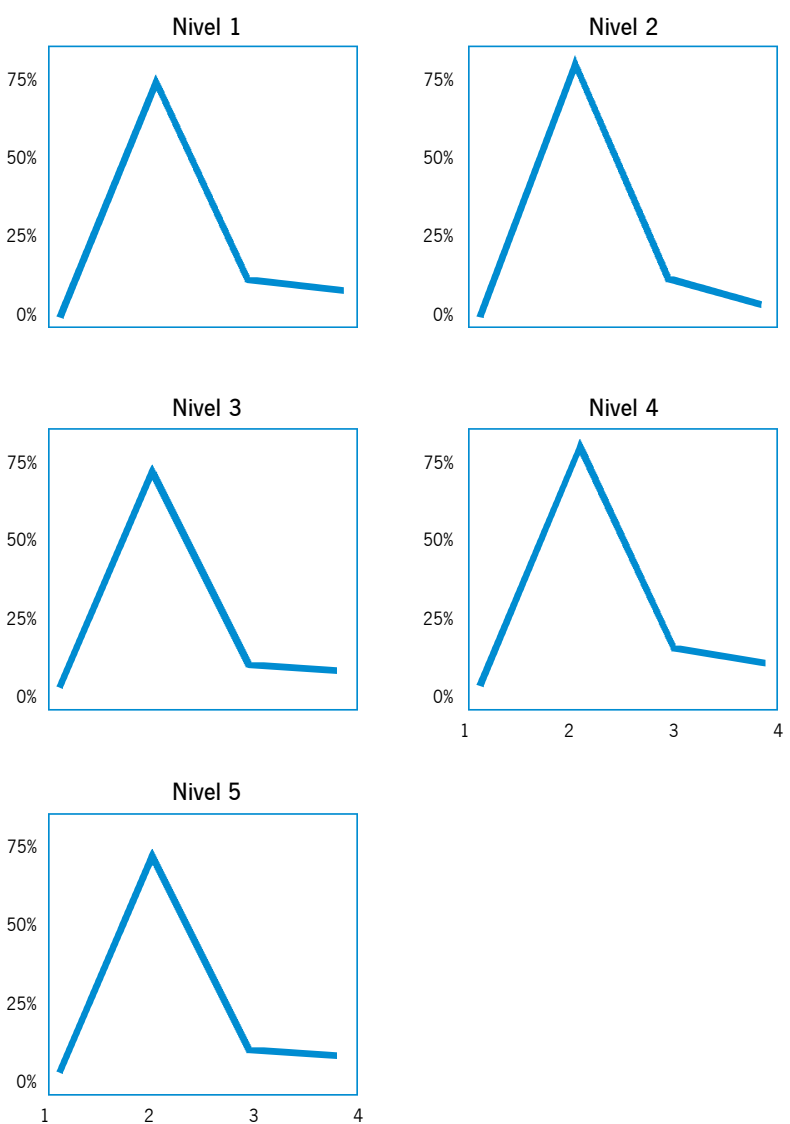

6. Nivel de agresividad en función de categorias de estado de conservación
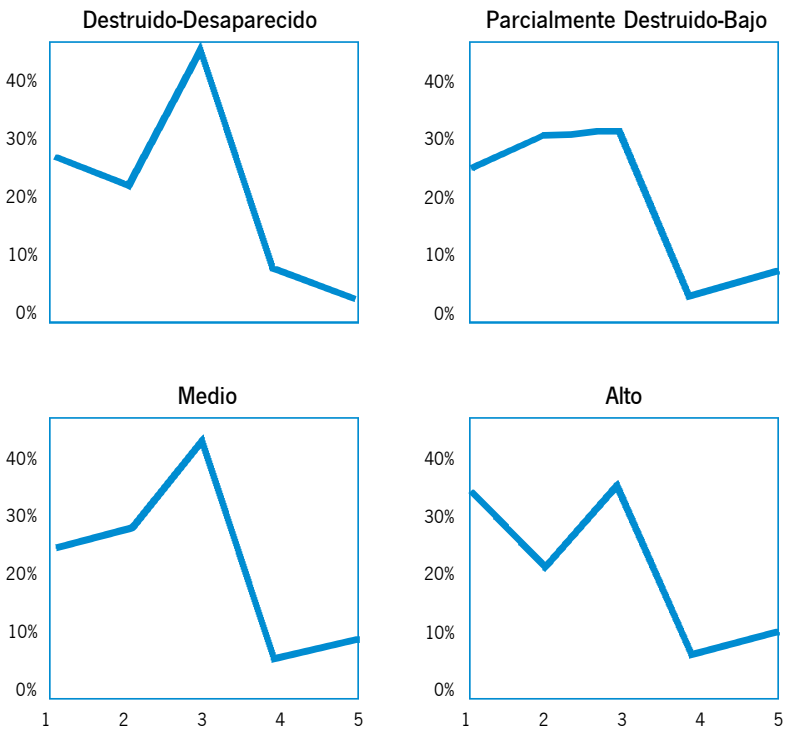

do tratamiento de los datos eliminando del análisis aquellos registros en los que no se especificaba el agente humano concreto, considerándolos valores perdidos del sistema, aumenta la medida de la asociación hasta un 0,64.

El examen de los porcentajes de las causas de deterioro en cada nivel de agresividad permiten destacar los siguientes aspectos:

a. Por un lado, para el nivel de agresividad 1 se aprecia un peso importante de la categoría "expolio superficial" $(24,1 \%)$ seguido de "arado superficial" (21,1\%). A falta de un análisis cruzado entre tipologías y/o periodos con nivel de agresividad, podemos intuir que determinadas EA, tales como conjuntos funerarios megalíticos, localizados muy frecuentemente en estos terrenos de quercineas y matorral arbolado, pueden llevarse el peso de esta tendencia observada.

Otra cuestión es el peso porcentual de "arado superficial". Las áreas asignadas a nivel 1 forman el dominio básico de pastizales, matorral y quercíneas, lo cual, en su forma de manejo humano, las podemos correlacionar a los dominios de dehesa. En estas áreas existe presencia de arado en tanto convivan la presencia de encina-alcornoque con áreas abiertas susceptibles de ser aprovechadas intensivamente como secano o pastos explotados y, por tanto, arables.

b. Para los niveles 2 y 3 se aprecia el fuerte peso porcentual del arado superficial, 49,1\% y 42,2,\% respectivamente, relacionado con los usos del suelo asignados previamente a estos niveles de agresividad.

c. Respecto a los niveles 4 y 5 se observa que el peso de la categoría "obras públicas" alcanza su mayor incidencia (18,8\%), así como las categorias "obras privadas" (11,3\%) y "desmontes" $(6,3 \%)$. En el nivel 5, destaca concretamente la relevancia de la categoría "labores forestales" (18,3\%).

En definitiva, las causas de deterioro por agentes humanos asignadas a cada EA parecen mostrar un buen ajuste a los niveles de agresividad inducidos desde los usos del suelo.

Grado de conservación y causas de deterioro en función del grado de protección

En los análisis preliminares realizados con la información contenida en DatARQUEOS (Fernández Cacho-Mondéjar-Díaz Iglesias, 2002) respecto a las variables tenidas en consideración en este apartado, se concluyó que el grado de conservación de las EAs situadas dentro de espacios naturales protegidos no difería de forma demasiado significativa con las localizadas fuera de ellos.

Sin embargo, se ha creido necesario profundizar en este análisis ya que a priori se presupone una mejor protección de los sitios arqueológicos en estos espacios y, sobre todo, en el caso de que hayan sido protegidos específicamente por la Consejería de Cul- 
tura, tanto porque la tutela administrativa es mayor, como porque se tienden a proteger sitios arqueológicos especialmente bien conservados.

En el primero de los casos se corroboran básicamente los resultados previos obtenidos: las EAs no ofrecen un estado de conservación significativamente mejor por estar situadas en un espacio natural protegido. Sin embargo se constatan diferencias apreciables en las causas de deterioro que afecta a unas y otras ya que en las ubicadas fuera de ellos, se documenta una mayor incidencia de los factores antrópicos en su estado de conservación.

Por otra parte, se observa cómo el estado de conservación de las EAs tiene una correlación positiva con su grado de protección a partir de los resultados obtenidos con la prueba del chi-cuadrado (Tabla 3. ver anexos). Sin embargo, si se visualizan en un gráfico los datos aportados por las tablas de contingencia se comprueba que esta asociación es muy débil, es decir, el valor asignado al estado de conservación de las EAs se distribuyen de forma homogénea independientemente de su grado de protección, hecho que constata la debilidad de la medida de asociación entre ambas $(0,156)$. En este caso, la negación de la independencia de las variables no significa necesariamente su dependencia real, ya que trabajando con un volumen de datos tan grande la independencia solo se afirmaría categóricamente en aquellos casos en los que los valores se distribuyeran exactamente igual.

\section{Conclusiones}

Los datos aportados por los análisis estadísticos realizados ofrecen unas medidas de asociación muy débiles entre las variables. El objetivo de obtener un modelo matemático de su comportamiento desde las fuentes de datos disponibles puede estar demandando análisis de cruce entre más de dos variables y, por tanto, la utilización de modelos logarítmicos.

Es importante destacar que los datos raramente pierden su validez y que éstos están condicionados por su modelado. Del modelo de datos de un sistema de información se extraen variables y habrá que preguntarse si su definición es válida o inválida, porque quizás uno de las principales aportaciones de este trabajo haya sido la constatación de que algunas categorías establecidas en las fuentes de información pueden estar restando valor informativo a determinadas variables.

Hay una falta de correlación significativa entre algunas de las variables seleccionadas, precisamente la que ofrece datos más genéricos (periodos históricos, tipologías, grado de protección y usos del suelo), y la información sobre el estado de conservación de las propias EAs, y que pueden verse condicionados por la subjetividad de quien recopila la información en el terreno. Sin embargo, las asociaciones establecidas entre el grado de agresivi- dad de los usos del suelo y los diferentes agentes humanos que han incidido en el estado de conservación de las EAs son más elevadas. Ello apunta a la necesidad de ampliar el módulo de conservación de la base de datos, que manifiesta su mayor utilidad en aquellos casos en los que se ha posibilitado el registro de información detallada.

Por otra parte, se propone la elaboración de los índices de perdurabilidad de sitios arqueológicos andaluces aplicando en mayor medida los métodos deductivos y no realizando inferencias a partir de los datos de conservación disponibles, aunque estos puedan complementar en algunos casos los resultados que se obtengan.

Por último, se sugiere la contrastación de dichos resultados mediante trabajos de campo en áreas concretas y representativas de las zonificaciones obtenidas en función de los índices de perdurabilidad propuestos.

\section{Bibliografía}

FERNÁNDEZ CACHO, S.; MONDÉJAR FERNÁNDEZ DE QUINCOCES, P.; DÍAZ IGLESIAS, J.M. (2002): "La información de Patrimonio Arqueológico en Andalucia: valoración general" En FERNÁNDEZ CACHO, S. (Ed.): ARQUEOS. Sistema de Información Arqueológico de Andalucia. Cuadernos Técnicos, 6. Granada: Instituto Andaluz del Patrimonio Histórico, Junta de Andalucía, pp. 143-165

MÁRQUEZ PÉREZ, J. y VALLEJO VILLALTA, I. (2004): Elaboración y cartografía de índices de perdurabilidad de sitios arqueológicos en Andalucía. Sevilla: Instituto Andaluz del Patrimonio Histórico. Informe inédito

MONDÉJAR FERNÁNDEZ DE QUINCOCES, P. (2002): "Fuentes y validación de la información de DatARQUEOS" En FERNÁNDEZ CACHO, S. (Ed.): ARQUEOS. Sistema de Información Arqueológico de Andalucía. Sevilla: Instituto Andaluz del Patrimonio Histórico, Junta de Andalucía, pp. 59-69

MUÑOZ REYES, A. (2003): Dictamen sobre la calidad de la información disponible para la ejecución del proyecto MAPA desde el punto de vista de su tratamiento estadístico. Sevilla: Instituto Andaluz del Patrimonio Histórico. Informe inédito

MUÑOZ REYES, A.M. y RODRIGO CÁMARA, J.M. (2004): Dictamen sobre la calidad de la información de conservación de entidades arqueológicas para la ejecución del proyecto 'Modelo Andaluz de Predicción Arqueológica desde el punto de vista de su tratamiento estadístico'. Sevilla: Instituto Andaluz del Patrimonio Histórico. Informe inédito

SHENNAN, S. (1992): Arqueología Cuantitativa. Barcelona: Editorial Crítica 


\section{$078-079$ \\ Proyectos del IAPH}

Los datos a examen:

estadística e IP de los sitios

arqueológicos andaluces

\section{PH49 - Julio 2004}

Tabla 1. Resultados del análisis de los valores de chi-cuadrado y de la intensidad de la relación

\begin{tabular}{|c|c|c|c|c|c|c|c|}
\hline \multirow[b]{2}{*}{ Estado de conservación } & \multirow[b]{2}{*}{$\begin{array}{l}\text { Periodo } \\
\text { Tipología genérico } \\
\text { Nivel de agresividad } \\
\text { Ubicación Espa. Prot. } \\
\text { Grado de protección }\end{array}$} & \multirow{2}{*}{$\begin{array}{l}\text { iasos válidos } \\
4645 \\
4044 \\
8389 \\
8389 \\
8389\end{array}$} & \multicolumn{3}{|c|}{ Chi-cuadrado } & \multicolumn{2}{|c|}{ Intensidad de la relación } \\
\hline & & & $\begin{array}{l}\text { Valor } \\
101.953 \\
356.629 \\
123.248 \\
32.357 \\
43.967\end{array}$ & $\begin{array}{l}\text { GI } \\
21 \\
66 \\
12 \\
3 \\
3\end{array}$ & $\begin{array}{l}\text { Significación } \\
0.000 \\
0.000 \\
0.000 \\
0.000 \\
0.000\end{array}$ & $\begin{array}{l}\text { Phi } \\
0.148 \\
0.297 \\
0.121 \\
0.062 \\
0.072\end{array}$ & $\begin{array}{l}\text { V de Cramer } \\
0.086 \\
0.171 \\
0.070 \\
0.062 \\
0.072\end{array}$ \\
\hline $\begin{array}{l}\text { Causas del deterioro } \\
\text { (genérico) }\end{array}$ & $\begin{array}{l}\text { Nivel de agresividad } \\
\text { Ubicación Espa. Prot. } \\
\text { Grado de protección }\end{array}$ & $\begin{array}{l}10696 \\
10696 \\
10696\end{array}$ & $\begin{array}{l}421.694 \\
167.980 \\
261.436\end{array}$ & $\begin{array}{l}4 \\
1 \\
1\end{array}$ & $\begin{array}{l}0.000 \\
0.000 \\
0.000\end{array}$ & $\begin{array}{l}0.199 \\
0.125 \\
0.156\end{array}$ & $\begin{array}{l}0.199 \\
0.125 \\
0.156\end{array}$ \\
\hline $\begin{array}{l}\text { Causas del deterioro } \\
\text { (ag. Natural) }\end{array}$ & $\begin{array}{l}\text { Nivel de agresividad } \\
\text { Ubicación Espa. Prot. } \\
\text { Grado de protección }\end{array}$ & $\begin{array}{l}3765 \\
3765 \\
3765\end{array}$ & $\begin{array}{l}205.561 \\
149.390 \\
93.670\end{array}$ & $\begin{array}{l}16 \\
4 \\
4\end{array}$ & $\begin{array}{l}0.000 \\
0.000 \\
0.000\end{array}$ & $\begin{array}{l}0.234 \\
0.199 \\
0.158\end{array}$ & $\begin{array}{l}0.117 \\
0.199 \\
0.158\end{array}$ \\
\hline $\begin{array}{l}\text { Causas del deterioro } \\
\text { (ag. Humano) }\end{array}$ & $\begin{array}{l}\text { Nivel de agresividad } \\
\text { Ubicación Espa. Prot. } \\
\text { Grado de protección }\end{array}$ & $\begin{array}{l}9861 \\
9861 \\
9861\end{array}$ & $\begin{array}{l}2082.780 \\
404.215 \\
969.783\end{array}$ & $\begin{array}{l}76 \\
19 \\
19\end{array}$ & $\begin{array}{l}0.000 \\
0.000 \\
0.000\end{array}$ & $\begin{array}{l}0.460 \\
0.202 \\
0.314\end{array}$ & $\begin{array}{l}0.230 \\
0.202 \\
0.314\end{array}$ \\
\hline
\end{tabular}

Tabla 2. Deterioro por agente humano. Porcentajes de causas y de nivel de agresividad

\section{$\%$ de causas de deterioro por agente humano}

Causas de deterioro por agente humano

Total
Agente humano sin especificar

Expolio superficial

Expolio con movimientos de tierra $<50 \%$

Expolio con movimientos de tierra $>50 \%$

Arado superficial

Arado subsolador

Puesta en riego

Labores forestales

Actividades extractivas

Labores industriales

Obras públicas

Dragados

Obras privadas

Excavaciones arqueológicas sin conservación

Régimen de visitas

Otros

Actividades pecuarias

Usos militares

Desmontes

Aterrazamientos
1

$\begin{array}{lll}33.6 \% & 30.1 \% & 33.0 \%\end{array}$

$47.8 \%-26.1 \%-23.2 \%$

$12.5 \% \quad 42.2 \% \quad 43.4 \%$

$\begin{array}{lll}7.3 \% & 33.7 \% & 57.5 \%\end{array}$

$\begin{array}{lll}10.0 \% & 29.4 \% & 56.3 \%\end{array}$

$35.4 \% \quad 17.5 \% \quad 17.5 \%$

$26.0 \% \quad 26.0 \% \quad 39.8 \%$

$53.8 \% \quad 15.4 \% \quad 30.8 \%$

$21.5 \% \quad 25.1 \%$

$14.3 \% \quad 28.6 \%$

$20.5 \% \quad 28.5 \%$

$55.0 \% \quad 21.7 \%$

$48.5 \% \quad 21.2 \%$

$30.2 \% \quad 23.8 \%$

$69.8 \% \quad 11.6 \%$

$66.7 \% \quad 16.7 \%$

$19.4 \% \quad 31.1 \%$

$32.5 \% \quad 25.0 \%$

$22.6 \%$

$32.8 \%$

$30.8 \%$
$44.4 \%$

$44.4 \%$
$47.6 \%$

$45.5 \%$

$20.0 \%$

$39.2 \%$

$14.0 \%$

$16.7 \%$

$43.4 \%$

$33.8 \%$

$39.2 \%$

4

5

Total

$4.6 \% \quad 15.6 \%$

$1.1 \% \quad 3.2 \%$

$1.7 \% \quad 2.3 \%$

$0.7 \%$

$0.7 \%$
$0.3 \%$

$0.3 \% \quad 1.2 \%$

$1.3 \% \quad 3.1 \%$

$2.7 \% \quad 27.0 \%$

$0.5 \% \quad 7.7 \%$

$3.6 \% \quad 5.4 \%$

$\begin{array}{ll}3.6 \% & 5.4 \% \\ 4.8 \% & 4.8 \%\end{array}$

$3.5 \% \quad 2.0 \%$

$1.7 \%$

$12.1 \% \quad 18.2 \%$

$2.6 \% \quad 4.1 \%$

$-\quad 4.7 \%$
$-\quad 6 \%$

$\begin{array}{ll}- & - \\ 4.1 \% & 2.0 \%\end{array}$

$1.8 \%$

$1.5 \% \quad 3.9 \%$

$100 \%$

$100 \%$

$100 \%$

$100 \%$

$100 \%$
$100 \%$
$100 \%$

$100 \%$

$100 \%$

$100 \%$

$100 \%$

$100 \%$

$100 \%$

$100 \%$

$100 \%$

$100 \%$

$100 \%$

$100 \%$

$100 \%$

$\%$ de nivel de agresividad

Causas de deterioro por agente humano

Agente humano sin especificar

Expolio superficial

Expolio con movimientos de tierra $<50 \%$

Expolio con movimientos de tierra $>50 \%$

Arado superficial

Arado subsolador

Puesta en riego

Labores forestales

Actividades extractivas

Labores industriales

Obras públicas

Dragados

Obras privadas

Excavaciones arqueológicas sin conservación

Régimen de visitas

Otros

Actividades pecuarias

Usos militares

Desmontes

Aterrazamientos

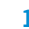

1

$12.8 \%$

$6.4 \% \quad 3.3 \%$

$3.0 \% \quad 1.1 \%$

$21.1 \% \quad 49.1 \%$

$3.0 \% \quad 9.6 \%$

$\begin{array}{ll}0.7 \% & 1.5 \% \\ 4.2 \% & 1.4 \%\end{array}$

$\begin{array}{ll}.2 \% & 1.4 \% \\ 0.3 \% & 1.6 \%\end{array}$

$0.3 \% \quad 0.1 \%$

4.8\% $\quad 3.9 \%$

$0.1 \% \quad 0.2 \%$

$\begin{array}{ll}3.6 \% & 3.5 \% \\ 1.5 \% & 0.4 \%\end{array}$

$0.7 \% \quad 0.2 \%$

$4.7 \% \quad 2.5 \%$

$1.3 \% \quad 0.2 \%$

$0.4 \% \quad 0.1 \%$

$\begin{array}{ll}0.7 \% & 1.9 \% \\ 3.3 \% & 1.8 \%\end{array}$

$3.3 \%$

$100 \%$

$100 \%$

\section{4}

$19.1 \%$
$11.2 \%$

$11.2 \%$
$3.9 \%$

-

$18.4 \%$

$2.0 \%$

$1.3 \%$
$4.6 \%$

$0.7 \%$
-
$11.8 \%$

$11.8 \%$

$0.7 \%$

$9.2 \%$

$0.7 \%$

$2.6 \%$

$5.9 \%$

- $\quad 0.5 \%$

$5.3 \% \quad 1.0 \%$

$2.6 \% \quad 4.1 \%$ 
Anexos. Tablas 1,2 y 3

Tabla 3. Grado de protección y grado de conservación. Correlación a partir de las pruebas de chi-cuadrado

$\begin{array}{lcccc} & \text { Pruebas de chi-cuadrado } & \text { gl } & \\ & \text { Valor } & 3 & 0 \\ \text { Chi-cuadrado de Pearson } & 43.967^{*} & 3 & 0 \\ \text { Razón de verosimilitud } & 46.16 & 1 & 0 \\ \text { Asociación lineal por lineal } & 37.595 & & \\ \text { № de casos válidos } & 8389 & \text { bilateral) }\end{array}$

*.0 casillas $(0.0 \%)$ tienen una frecuencia esperada inferior a 5 . La frecuencia mínima esperada es de 12.44

\section{Medidas simétricas}

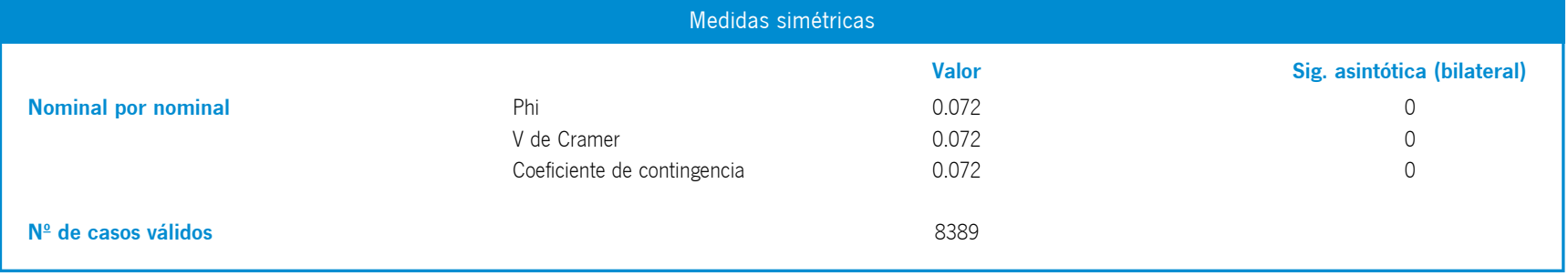

\begin{tabular}{|c|c|c|c|c|c|c|}
\hline \multicolumn{7}{|c|}{ \% de Grado de protección } \\
\hline \multirow{3}{*}{ Grado de protección } & & Destruido-Desaparecido & Parcialmente Destruido-Bajo & Medio & Alto & Total \\
\hline & Protegido & $0.2 \%$ & $70.5 \%$ & $19.2 \%$ & $10.1 \%$ & $100 \%$ \\
\hline & No protegido & $1.6 \%$ & $78.1 \%$ & $13.4 \%$ & $6.8 \%$ & $100 \%$ \\
\hline Total & & $1.5 \%$ & $77.4 \%$ & $14.0 \%$ & $7.2 \%$ & $100 \%$ \\
\hline
\end{tabular}
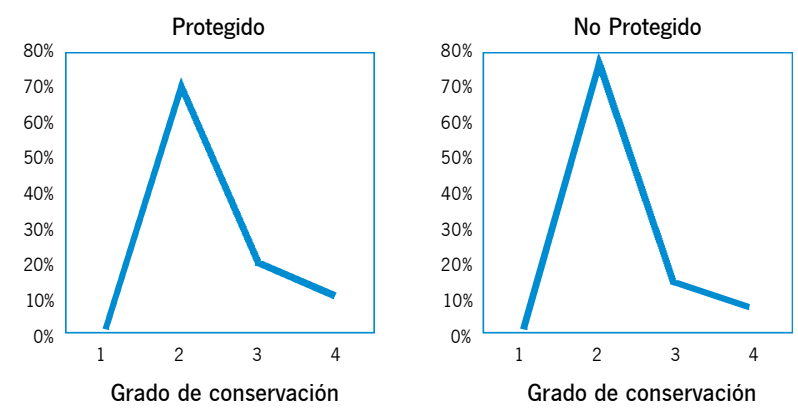

\begin{tabular}{|c|c|c|c|c|c|c|}
\hline \multicolumn{7}{|c|}{$\%$ de Grado de conservación } \\
\hline \multirow{3}{*}{ Grado de protección } & & Destruido-Desaparecido & Parcialmente Destruido-Bajo & Medio & Alto & Total \\
\hline & Protegido & $1.6 \%$ & $9.1 \%$ & $13.6 \%$ & $14.0 \%$ & $10 \%$ \\
\hline & No protegido & $98.4 \%$ & $90.9 \%$ & $86.4 \%$ & $86.0 \%$ & $90 \%$ \\
\hline Total & & $100.0 \%$ & $100.0 \%$ & $100.0 \%$ & $100.0 \%$ & $100.0 \%$ \\
\hline
\end{tabular}
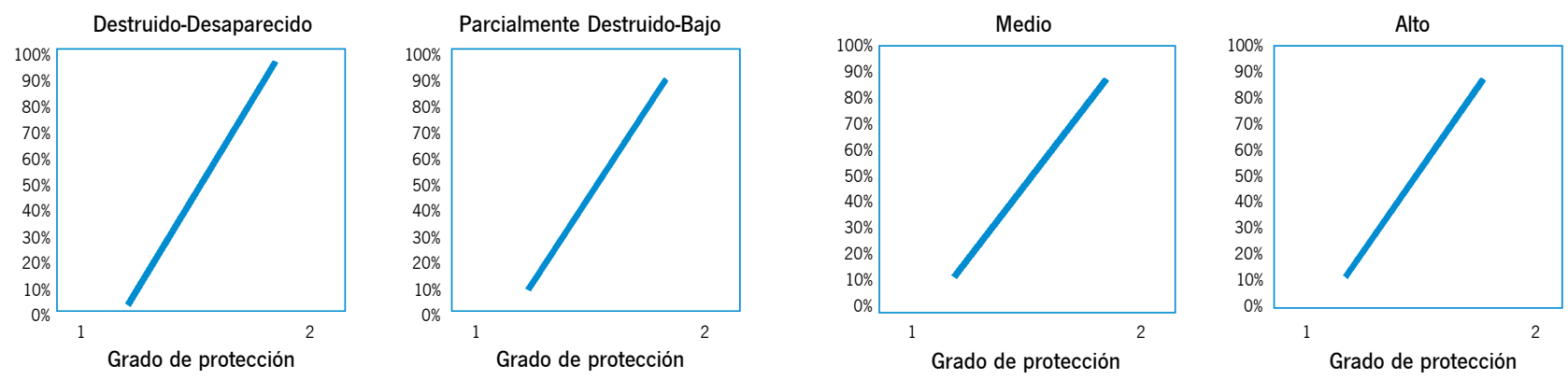